\title{
VoIP performance over Mobile WiMAX: An Urban Deployment Analysis
}

\author{
João Henriques, Vitor Bernardo, Paulo Simões, Marilia Curado \\ Center for Informatics and Systems, \\ University of Coimbra \\ Polo II, Pinhal de Marrocos, \\ 3030-290 Coimbra, Portugal \\ jpch@student.dei.uc.pt, \{vmbern, psimoes, marilia\}@dei.uc.pt
}

\begin{abstract}
The significant growing of Voice over IP (VoIP) ready mobile devices raises new challenges in the deployment of novel broadband wireless access networks (BWA), such as WiMAX or Long Term Evolution (LTE). Due to voice service importance for the mobile market, the empirical assessment of voice traffic performance and quality is crucial for a successful deployment. In this work, the Mobile WiMAX (IEEE 802.16e) capabilities to support VoIP traffic under different scenarios and employing distinct Quality of Service (QoS) service classes were performed. Additionally, the paper characterizes the heterogeneity access conditions within a city, by analyzing both Line of Sight (LOS) and Non-Line of Sight (NLOS) conditions. By examining the end-user perceived quality (Quality of Experience) and the network QoS related parameters, the attained results shown the impact of the correct WiMAX QoS service classes management on the number of well served VoIP users.
\end{abstract}

Keywords-Mobile WiMAX; VoIP; Testbed; Quality of Service; Quality of Experience; IEEE 802.16e

\section{INTRODUCTION}

Future Internet will encompass an increasing number of users, aiming to be always best connected [1] anytime and anywhere. Besides network availability, application performance should also be taken into account, particularly when using multimedia applications, such as Voice over IP (VoIP) or Video Streaming. Novel broadband wireless access technologies, such as Worldwide Interoperability for Microwave Access (WiMAX) or Long Term Evolution (LTE), are being developed aiming to accomplish these goals. The exhaustive usage of the 4G and beyond "all-IP" broadband wireless access technologies, where all the traffic will be managed as an IP packet, raise new issues related with end-user quality perception.

By using an urban real Mobile WiMAX deployment in the city of Coimbra, Portugal, this work performs an empirical evaluation of VoIP performance over Mobile WiMAX, performing experiments in different access scenarios, namely in Line of Sight (LOS) and Non-Line of Sight (NLOS) conditions. The assessment includes also the study of Mobile WiMAX native Quality of Service (QoS) mechanisms through the usage of different QoS service classes, and a scalability / performance tradeoff analysis when supporting several simultaneous VoIP calls. Besides analyzing the network typical QoS parameters, such as packet loss rate or jitter, this work also performs a Quality of Experience (QoE) assessment of each VoIP call, aiming to comprehend the impact of the studied network conditions and scenarios in the end-user perceived quality.

The rest of the paper is organized as follows: Section II gives a brief description of the WiMAX technology, and presents the main motivations of this work. Section III discusses the most significant related work on WiMAX assessment. In Section IV the testing methodology and the testbed configuration for the VoIP evaluation are described. The results obtained in the Mobile WiMAX testbed are presented in Section V. Finally, Section VI presents the conclusions and future work.

\section{BACKGROUND AND MOTIVATION}

WiMAX and LTE are both broadband wireless access (BWA) network technologies, aiming to play a crucial role in $4 \mathrm{G}$ and beyond "all-IP" broadband wireless access technologies progress. LTE is an evolved 3GPP technology [2], and as WiMAX, was developed to support higher number of users with higher data rates, coverage and availability. However, LTE is not in the scope of this work, which focuses on the WiMAX technology. This technology is based on the IEEE 802.16 standards [3], and the most relevant versions are the Fixed WiMAX, based on IEEE 802.16d [4], and the Mobile WiMAX, defined by IEEE 802.16e [5]. The latter has significant improvements in the support of multiple users, as well as new mechanisms for Quality of Service, mobility support and also energy efficiency. This technology is planned to achieve long ranges of coverage with high availability and throughput, allowing rural or urban wireless access in diverse deployment environments, namely with and without line of sight.

The QoS support is provided by different service classes, allowing the traffic flows differentiation by setting the applicable network parameters, namely the maximum and minimum reserved traffic rate, the maximum allowed delay, or the maximum tolerable jitter. The service classes ordered by traffic prioritization (higher to lower) are the following: Unsolicited Grant Service (UGS), Extended Real Time Polling Service (ertPS), Real Time Polling Service (rtPS), Non-Real Time Polling Service (nrtPS), and Best Effort (BE). Each QoS 
service class is suitable to different applications (e.g. rtPS is befitting for real-time applications and nrtPS performs better for bulk file transfers).

The WiMAX QoS model is based on service flows. Each service flow is a unidirectional flow, with QoS parameters defined to fulfill the application requirements, to which is assigned a connection, identified by a connection identifier (CID). Each node can have multiple connections and service flows. The Base Station (BS) always manages the traffic scheduling for the different service classes, whether in the uplink or downlink. Another relevant feature of Mobile WiMAX is the support of energy saving mechanisms, such as the idle and sleep modes. The idle mode allows the node to be completely turned off and deregistered from the base stations for certain periods, consuming less energy, while the sleep mode disrupts the nodes connections individually, needing normal handoff procedures, and so, consuming more energy than the idle mode. These energy efficiency mechanisms are particularly relevant in the context of mobile networks, since the majority of connected devices are battery based.

It should be noted that the technology supports different access scenarios, namely fixed, nomadic and mobile. WiMAX technology supports full mobility, allowing the access to the network at moving speeds, supporting vertical seamless handovers, where the users do not notice the attachment point change.

The main goal of this work is to assess the WiMAX technology capabilities to support VoIP traffic in a real deployment urban testbed, within LOS and NLOS scenarios, using different native WiMAX QoS mechanisms. This empirical study is focused on the assessment of the end-user perceived quality, also known as QoE, in a multi-user environment performing real VoIP calls, emulated by simultaneous bi-directional VoIP traffic flows.

\section{RELATED WORK}

This section presents the most relevant related work on WiMAX assessment.

Oh et al. [6] evaluate distinct WiMAX QoS mechanisms using Automatic Repeat Request (ARQ) in a simulation environment using OPNET [7]. In this work the VoIP traffic assessment was performed with special emphasis on the uplink scheduling mechanisms of WiMAX. The study encompasses the usage of three different WiMAX QoS classes, namely ertPS, rtPS and UGS. The results demonstrate that the usage of ARQ in ertPS can save wireless resources, while the usage of this mechanism in UGS causes deterioration in the perceived VoIP quality.

A study of voice traffic over Mobile WiMAX using both LOS and NLOS conditions was performed by Zhang et al. [8]. The evaluation procedure was performed during the handover (Hard Hand Over (HHO)) in order to assess the impact of this procedure during a voice call. The network parameters evaluated are the jitter, the packet loss and the delay. The Quality of Experience, assessed using the Perceptual Evaluation of Speech Quality (PESQ), was obtained by sending an audio file from the sender to receiver. Then, an analysis of the audio file is conducted, resulting in a value between 1 and 4.5, representing the end user perceived quality. The tests are performed without specifying a channel with QoS (i.e., using a BE channel). This work verified that when the node is at the cell-center with LOS the PESQ values are higher, when compared to the cell-edge NLOS values. Furthermore, it was also showed that performing $\mathrm{HHO}$ has a direct impact in the packet loss, and consequently, in the enduser perceived quality.

Jadhav et al. [9] evaluate, using the OPNET simulator, the differences between WiMAX and Universal Mobile Telecommunications System (UMTS) when transmitting voice data to multiple users simultaneously, employing the G.711 codec. The channel configuration used is $\mathrm{BE}$ for both technologies. This work evaluates network QoS parameters such as jitter, delay and packet loss, but also the QoE perceived by the end user, through the MOS. This evaluation is limited to a low number of simultaneous users and the main focus is on the comparison between WiMAX and UMTS, leaving behind the comparison between different QoS service classes. WiMAX has proven to have better capabilities while supporting VoIP applications, allowing a higher number of simultaneous users within a good perceived quality.

Durantini et al. [10] evaluated the WiMAX performance and capabilities in a real Fixed WiMAX testbed when transmitting Video on Demand (VoD), video streaming and web traffic with different QoS service classes, within different LOS and NLOS conditions in fixed and nomadic scenarios. Additionally, it also assesses the difference between different modulation schemes. These measurements are focused only in QoS metrics, such as delay and throughput. The results demonstrated that higher throughputs were achieved with more complex modulation schemes and that the rtPS is the most suited service class for video transmissions.

Bernardo et al. [11] present VoIP traffic evaluation over a real WiMAX tested using different transport protocols, such as User Datagram Protocol (UDP) / Real-time Transport Protocol (RTP) and Datagram Congestion Control Protocol (DCPP) in overestimated and underestimated scenarios. From the results obtained, it is shown that in overestimated scenario the One Way Delay (OWD) and packet loss have higher values when using DCCP protocol than with UDP. MOS also decreases, since it is related to the OWD and packet loss. On underestimated scenarios, the behavior when using DCCP is better than in the overestimate scenario. Nonetheless, it is always worst than when using UDP, which support higher MOS values. Although this evaluation also assesses the endto-end delay, packet loss and user perceived MOS, it is only performed over an rtPS configured channel.

Table I summarizes the discussed related work. The "Type" indicates the environment used in the tests, and the WiMAX related fields, "Version" and "QoS Service Classes", show the used WiMAX version and the native WiMAX QoS reservation channel employment during the tests. Finally, the "Assessment" related fields depict the used metrics during for the performed evaluations. 
TABLE I. RELATED WORK SUMMARY

\begin{tabular}{|c|c|c|c|c|c|}
\hline \multirow{2}{*}{ Work } & \multirow{2}{*}{ Type } & \multicolumn{2}{|c|}{ WiMAX } & \multicolumn{2}{c|}{$\begin{array}{c}\text { Assessment } \\
\text { Metrics }\end{array}$} \\
\cline { 3 - 6 } & Version & $\begin{array}{c}\text { QoS } \\
\text { Service } \\
\text { Classes }\end{array}$ & QoS & QoE \\
\hline $\begin{array}{c}\text { Oh et al. } \\
\text { [6] }\end{array}$ & Simulation & Mobile & Yes & Yes & Yes \\
\hline $\begin{array}{c}\text { Zhang et } \\
\text { al. [8] }\end{array}$ & Testbed & Mobile & No & Yes & Yes \\
\hline $\begin{array}{c}\text { Jadhav et } \\
\text { al. [9] }\end{array}$ & Simulation & Mobile & No & Yes & Yes \\
\hline $\begin{array}{c}\text { Durantini } \\
\text { et al. [9] }\end{array}$ & Testbed & Fixed & Yes & Yes & No \\
\hline $\begin{array}{c}\text { Bernardo } \\
\text { et al. [11] }\end{array}$ & Testbed & Fixed & Yes & Yes & Yes \\
\hline $\begin{array}{c}\text { This } \\
\text { Work }\end{array}$ & Testbed & Mobile & Yes & Yes & Yes \\
\hline
\end{tabular}

From the works described, none has an analysis of the enduser perceived voice quality while comparing different WiMAX QoS service classes, using a Mobile WiMAX real deployment. This work aims to fill this gap, in a real Mobile WiMAX testbed deployed in a city environment. This assessment will also evaluate the differences between some of the available QoS service classes, and both LOS and NLOS scenarios, using real end-user equipment.

\section{Methodology}

In this section the several aspects of the tests specification and methodologies, as well as the testbed configuration and equipment used are presented.

\section{A. Testbed Configuration}

This work was performed on a Mobile WiMAX (IEEE 802.16e) real deployment testbed, consisting of two BSs Alvarion BreezeMax Macro Outdoor connected to the same Access Service Network (ASN)-Gateway - Alvarion BreezeMax ASNGW Mini-Centralized - through a centralized architecture, making the testbed fully compliant with the WiMAX Forum Network Reference Model (NRM) [12]. The testbed comprehends two Base Stations, but only the one providing connectivity in urban conditions was used in this assessment. Each BS is equipped with two Dual-Slant antennas ( $65^{\circ}$ for each sector, with dual polarization).

The BS is configured with $4 \times 2$ Multiple Input Multiple Output (MIMO) Matrix A (allowing better coverage, i.e., both antennas send the same data stream), operating in $2.615 \mathrm{GHz}$, with a $10 \mathrm{MHz}$ channel bandwidth. This channel is configured with Adaptive Modulation and Coding (AMC), allowing different modulation schemes for different clients. Each client may modify the modulation scheme due to weather conditions, line of sight constrains or distance to the BS. As the communication mechanism is Time Division Duplex (TDD), the frames are divided to the uplink and downlink communications. In this testbed, the frame configuration is $60 \% / 40 \%$, where $60 \%$ is for downlink and $40 \%$ is for uplink. The equipment configuration was the same in all the tests performed. In order to assess LOS and NLOS scenarios, two distinct locations were used and named correspondingly, LOS and NLOS. These locations are shown in Figure 1.

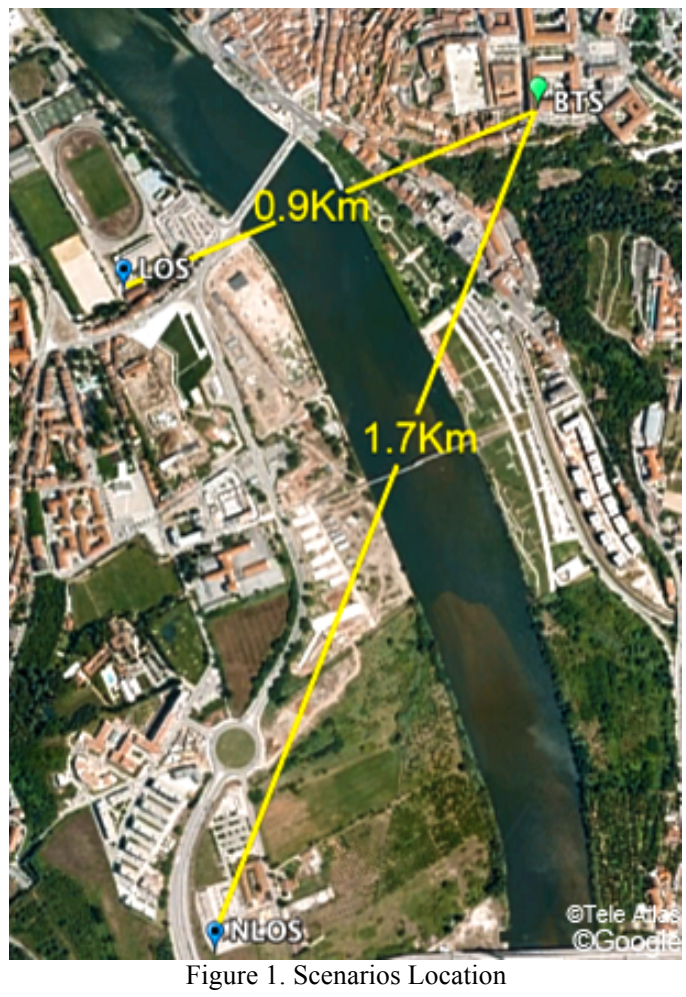

Both locations are within an urban scenario. The one without line of sight (NLOS) is located $1.7 \mathrm{Km}$ away from the BS. This location was used since it represents a good testing location for NLOS urban conditions, where the node is connected to the network behind buildings and where the signal is only obtained through reflections (i.e., line of sight to the BS is not actually possible). The one with line of sight (LOS) is located $0.9 \mathrm{Km}$ away from the BS, allowing near-optimal conditions for the nodes (close to the BS, clear line of sight).

All the tests were performed with mobile nodes, using two netbooks equipped with an USB Customer Premises Equipment (CPE). In the core network, a fixed node was used, connected to the network via Ethernet. All the nodes were running Debian Linux. The testbed architecture is illustrated in Figure 2.

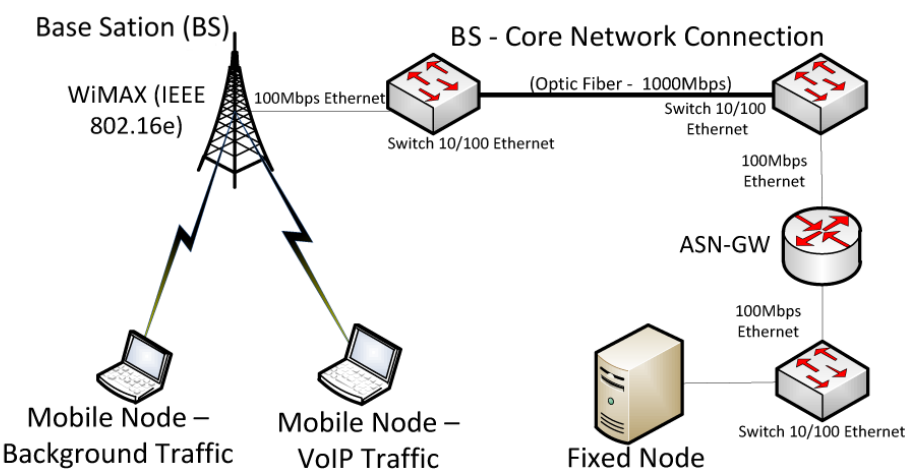

Figure 2. IEEE 802.16e Testbed Architecture 


\section{B. Tests Specification}

To perform the WiMAX technology capabilities assessment, a set of scripts and applications were needed. Based on the output network parameters and in the related work, the D-ITG [13] tool was used, since it allows different types of emulated traffic (e.g., VoIP with G.711.1 CODEC). D-ITG is able to generate realistic traffic patterns, and also provides an analysis of the most common network parameters, such as jitter, delay, and packet loss. The packet rate of voice data was 100 packets per second, with a compression rate of $96 \mathrm{Kbps}$, as defined by CODEC G.711.1 [14]. The number of simultaneous flows was: 1, 5, 10, 20, 30, 40, 50 and 60 flows, where each flow is intended to emulate one real bi-direction call (i.e., one user). For all the tests the same traffic generator seed was used, in order to generate similar traffic patterns across the tests. Each test is repeated through 5 runs to avoid the outlier results caused by signal fluctuations and unpredictable changes in the environment, since it is a real deployed testbed. The standard deviation is represented by vertical lines in graphs, calculated with a confidence interval of $95 \%$. The flows were started incrementally, and the measured period was when all flows were active. This methodology was used to avoid packet loss at the sender side, caused by buffer overflows.

All the VoIP traffic was transmitted over an rtPS or a BE channel. Initially, this communication was planned to be over an ertPS or UGS channel, which are best suited for VoIP traffic, but due to Alvarion hardware/software limitations, it was not possible to use these mechanisms. These limitations are caused when setting the maximum reserved rate to high values (in order to allow a large number of flows), and, since this service class is only prepared for low bandwidth usage (voice data), it was not possible to emulate more than 10 simultaneous voice calls.

The usage of rtPS mechanism was due to its guarantees, such as maximum tolerated OWD, reserved rate and traffic prioritization. The maximum tolerated OWD was set to $150 \mathrm{~ms}$, as recommended in ITU-T Y.1541 [15] and by WiMAX Forum [16]. The reserved rate was overestimated, in order to guarantee a higher rate than needed by all the flows, defined as $15 \mathrm{Mbps}$. BE was used with the objective of evaluating the real differences between both service classes, where the reserved rate for this class was also $15 \mathrm{Mbps}$. In order to complement this analysis, these tests were also carried out with background traffic. Using the tool IPerf [17], random traffic was emulated to represent a channel obstructed with data from other users. This traffic was generated in a BE channel, using all the bandwidth allowed by the BS. Therefore, the main goal was to evaluate the efficiency of native QoS mechanisms of WiMAX, observing the behavior of VoIP traffic when the channel is obstructed.

\section{Evaluation Metrics}

This section describes the main evaluation metrics used in the following tests, describing each one by categories: Quality of Service and Quality of Experience.

\section{1) Quality of Service}

The network QoS metrics assessed during this work are the delay and packet loss. The delay is measured through the Round Trip Time (RTT). As the mobile nodes are connected to the network only through WiMAX and they are not equipped with Global Positioning System (GPS) cards, it was not possible to accurately synchronize them to enable the correct OWD measurement.

The packet loss rate assessment enables the comparison of the obtained results with the ITU-T Y.1541 Recommendation, where the acceptable ranges for the different network Quality of Service are defined. For instance, for VoIP applications the ITU-T Y.1541 defines $150 \mathrm{~ms}$ as the maximum acceptable OWD and a maximum packet loss of $1 \%$. These QoS metrics allow the evaluation of network conditions, however, they do not allow assessing the impact of those conditions in the endusers perceived quality. To overcome this limitation, Quality of Experience metrics were also employed, as explained in the next subsection.

\section{2) Quality of Experience}

The Quality of Experience perceived by the end user is the main focus of this work, and it can be measured through several metrics. The most commonly used metrics are the MOS, E-Model, PESQ [18] and Perceptual Evaluation of Audio Quality (PEAQ) [19]. Since the tests were intended to be non-intrusive, the MOS and E-Model [20] were employed. MOS consists of several users evaluating one service, giving a score of one to five, where one is the worst value and five is the best value. MOS is a subjective metric commonly used in voice applications. However, the E-Model overcomes the need of real users and the subjectiveness associated to this evaluation, calculating the R-Factor and associating it value to the MOS Scale. The E-Model evaluates the VoIP Quality of Experience through network parameters, such as OWD and packet loss rate. In Cole et al. [21] the authors have reduced the R-Factor calculation to an equation based on OWD and packet loss values. Those equations were used to perform the MOS calculations in this work.

It should be noted that the MOS values depend on several factors, not only the network parameters such as delay and packet loss, but also on the codec used. For the G.711.1 codec used in this work, based on the calculations and parameters provided, the maximum MOS value in optimal conditions is 4.43 [21]. Table II depicts the relationship between the enduser perceived quality and MOS values.

\begin{tabular}{|c|c|}
\multicolumn{1}{c}{ TABLE II. } & \multicolumn{2}{c|}{ MOS VALUES } \\
\hline MOS & Quality \\
\hline 5 & Excellent \\
\hline 4 & Good \\
\hline 3 & Fair \\
\hline 2 & Poor \\
\hline 1 & Bad \\
\hline
\end{tabular}




\section{EXPERIMENTAL ASSESSMENT}

In this section the main goals and objectives are explained, as well as the analysis of the results obtained and their description.

\section{A. Objectives}

This assessment aims to achieve three main goals. The first consists on the native WiMAX QoS mechanisms evaluation and their impact on the end-user QoE, using different WiMAX service classes, such as rtPS and BE. The second objective measure is the impact of different line of sight conditions in both QoS and QoE metrics. Finally, this empirical works aims to address the effect of background traffic in the end-user perceived quality, as well as in the WiMAX network global performance. The results are presented and discussed in the following subsections.

\section{B. LOS Scenario}

In this subsection the results obtained in LOS scenarios are shown and explained.

\section{1) Packet Loss without BG traffic}

Figure 3 depicts the packet loss without background traffic in LOS conditions. The packet loss values are comparable in both service classes.

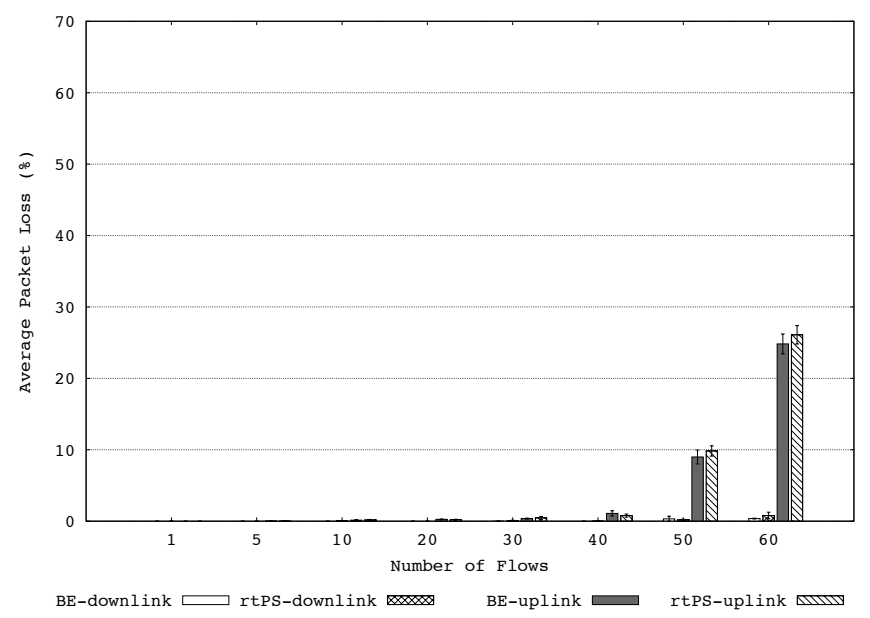

Figure 3. Packet Loss without background traffic in LOS conditions

In the uplink, the first noticeable impact of the packet loss is caused when transmitting more than 30 simultaneous flows (i.e., $<1 \%$, as advise in ITU-T Y.1541 recommendation). For instance, when transmitting 40 simultaneous flows, the uplink packet loss is $1 \%$ for $\mathrm{BE}$ and $0.8 \%$ for rtPS. With 50 simultaneous flows, these values are, respectively, $9 \%$ and $9.8 \%$ for BE and rtPS. In the downlink, the packet loss values remain below $1 \%$ with all simultaneous flows, for both service classes. From these results, it is possible to observe that the packet loss increases with the growth of the number of flows, since the bandwidth usage becomes higher.

The differences between uplink and downlink are due to USB stick transmission power limitations and the uplink/downlink testbed frame ratio configuration.

\section{2) Packet Loss with BG traffic}

Figure 4 depicts the packet loss with background traffic in LOS conditions. In this case, the maximum feasible packet loss limit (i.e., $<1 \%$ ) is achieved with 20 simultaneous flows. For 30 simultaneous flows, packet loss rate is already $4 \%$, which is $3 \%$ beyond the acceptable limit.

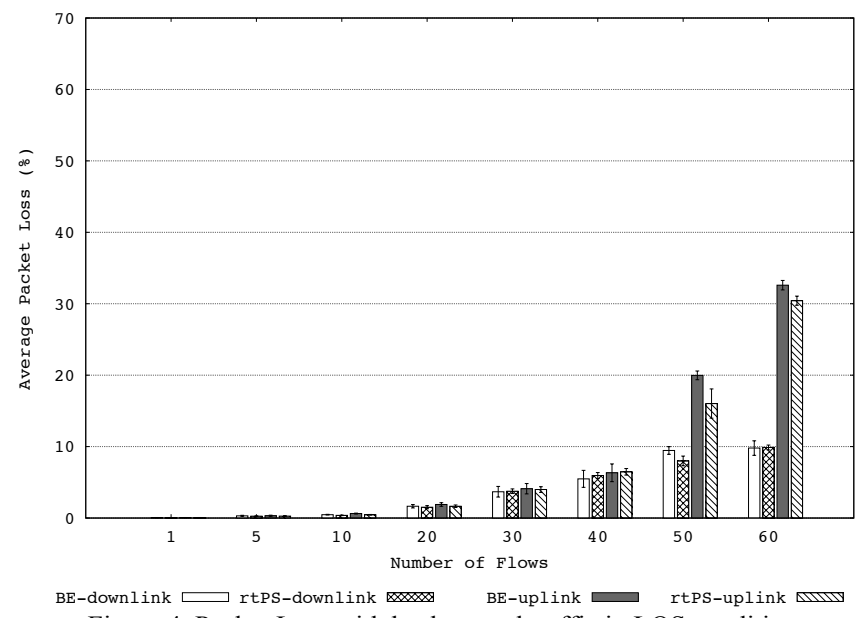

Figure 4. Packet Loss with background traffic in LOS conditions

The packet loss in the presence of background traffic is higher than when just VoIP traffic is being transmitted (Figure 3). These values are explained due to the high traffic load on the link, due the background traffic usage. The rtPS service class effectiveness is perceived, but only marginally.

3) MOS without BG traffic

Figure 5 shows the MOS without background traffic in LOS conditions.

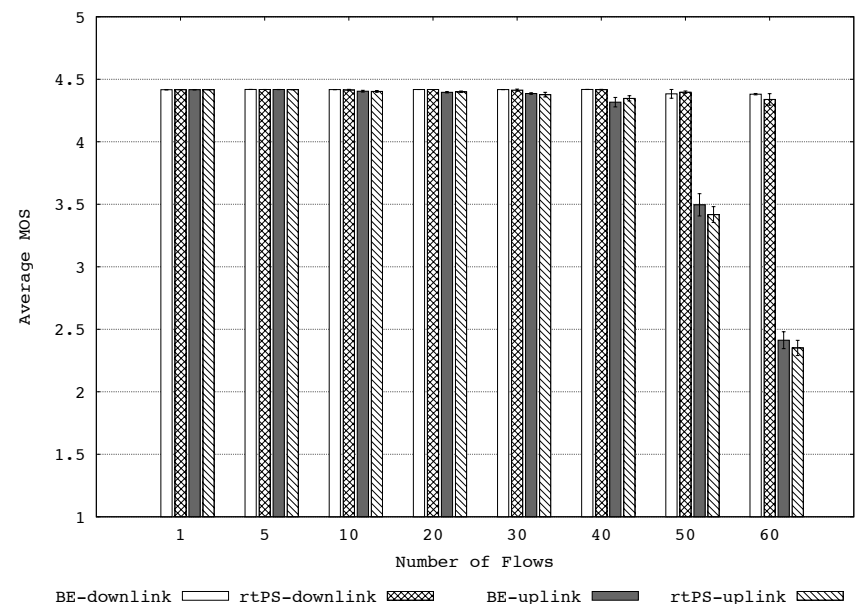

Figure 5. MOS without background traffic in LOS conditions

It demonstrates that without background traffic in LOS conditions it is possible to support up to 50 flows with "fair" quality (i.e., higher than 3). In this scenario, rtPS is slightly worse than BE, which can be caused by the QoS scheduling mechanisms overhead. 


\section{4) MOS with BG traffic}

The MOS with background traffic in LOS conditions is depicted in Figure 6. It is noticeable that the rtPS service class has some advantages when compared with BE. It guarantees "fair" quality up to the same 50 flows as without background traffic (Figure 5), while the BE service class only supports up to 40 simultaneous flows.

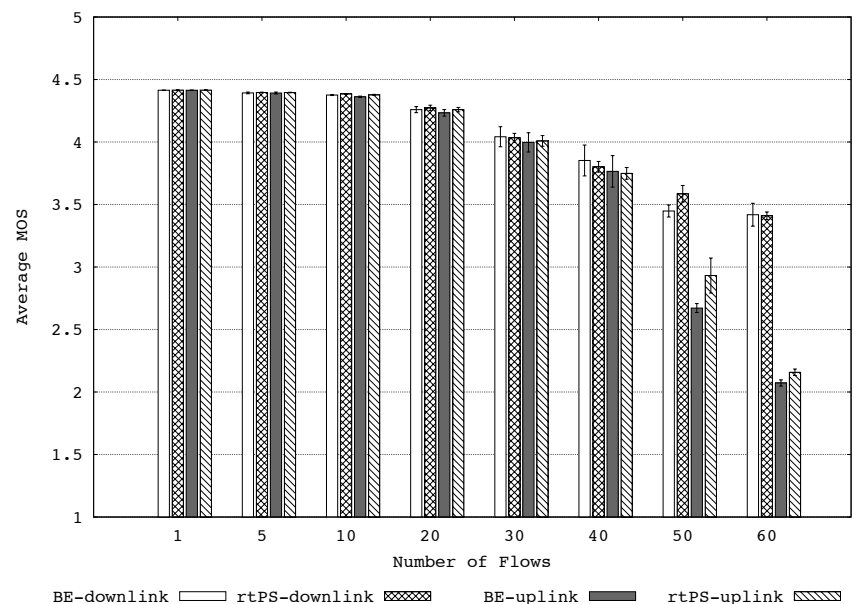

Figure 6. MOS with background traffic in LOS conditions

In Line of Sight conditions with background traffic, the differences between downlink and uplink are not so noticeable, since both communication directions are congested.

\section{NLOS Scenario}

This subsection presents the results concerning assessment performed in Non-Line of Sight conditions.

\section{1) Packet Loss without BG traffic}

Figure 7 shows the packet loss rate without background traffic in NLOS conditions.

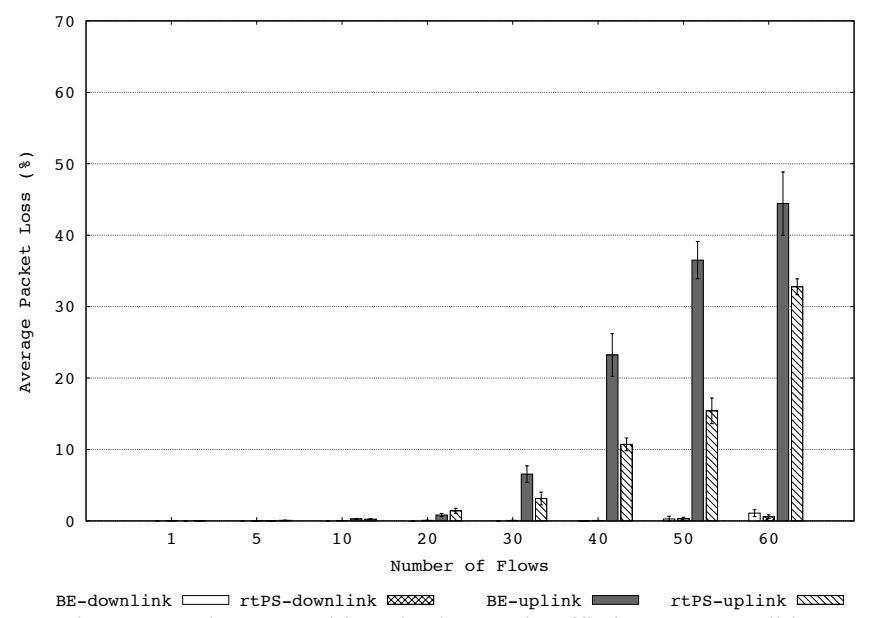

Figure 7. Packet Loss without background traffic in NLOS conditions

This figure shows that WiMAX supports 30 simultaneous flows under $10 \%$ for BE service class and 40 simultaneous flows under $10 \%$ for rtPS. In this scenario, for the same number of simultaneous flows, the packet loss rate is higher than in LOS scenario (Figure 3). This fact is caused by the lower bandwidth available due to the worst signal quality and the low CPE transmission power. Also, in this scenario, the differences between BE and rtPS are more noticeable.

\section{2) Packet Loss with BG traffic}

Figure 8 shows the packet loss percentage with background traffic in NLOS conditions. The differences between rtPS and $\mathrm{BE}$ are visible, but with minor differences.

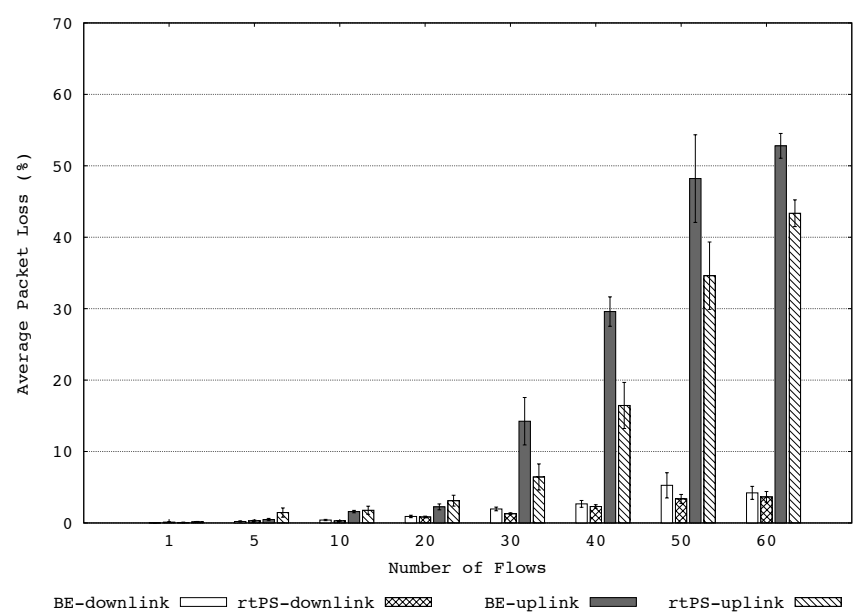

Figure 8. Packet Loss with background traffic in NLOS conditions

Figure 8 shows that in NLOS conditions with background traffic it is possible to support, under $10 \%$ of packet loss, up to 20 simultaneous flows for $\mathrm{BE}$ service class and 30 simultaneous flows for rtPS. This represents a lower capacity for supporting several flows when compared to Figure 4, where the packet loss values, for both uplink and downlink and rtPS and BE, were bellow $10 \%$ until 40 flows. The worst obtained values are in this scenario, as expected, since it represents the NLOS access conditions with a full load channel.

\section{3) MOS without BG traffic}

The Mean Opinion Score metric with background traffic in NLOS access condition is presented in Figure 9.

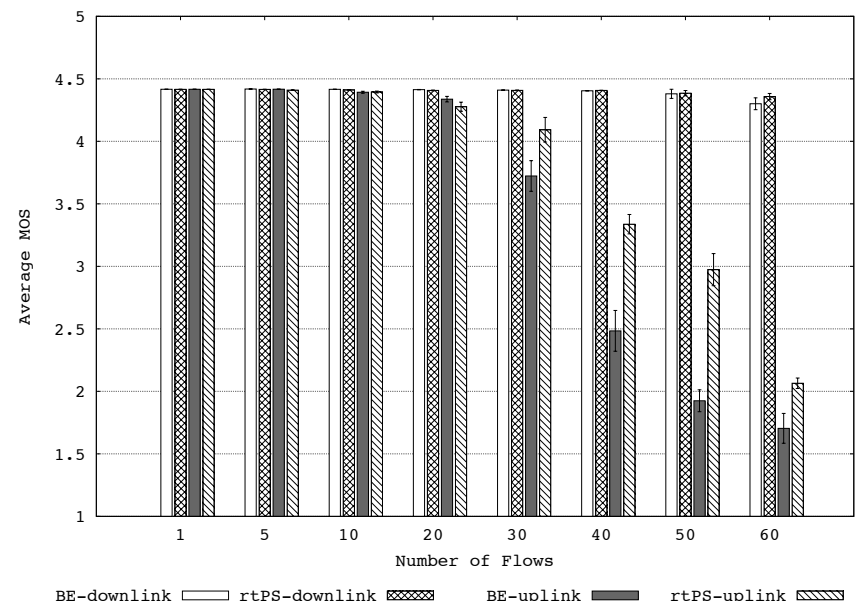

Figure 9. MOS without background traffic in NLOS conditions 
In this scenario, the WiMAX technology was able to support up to 50 simultaneous flows using the rtPS service class, while with BE only 30 simultaneous flows are sustained. This well sustained number assumes the expected "fair" conditions to the end-user perceived quality (i.e., QoE). When comparing the NLOS and LOS scenarios without background traffic, (Figure 5 and Figure 9) the MOS values start decreasing at 40 simultaneous flows in LOS, while in NLOS, it starts decreasing at 20 flows. In short, the rtPS service class supports, in both NLOS and LOS, up to 50 simultaneous flows with "fair" quality. The BE class supports up to 50 flows in LOS and, in NLOS, supports a maximum of 30 flows.

\section{4) MOS with BG traffic}

Figure 10 depicts MOS values with background traffic in NLOS conditions. In this scenario, WiMAX supports up to 40 flows in the rtPS service class, while in BE it supports up to 30 simultaneous flows (with "fair" conditions). This represents a decrease of 10 flows for rtPS, when compared to the previous scenario, without background traffic. Also, the differences between rtPS and BE are clearly shown, where rtPS supports more 10 flows than $\mathrm{BE}$.

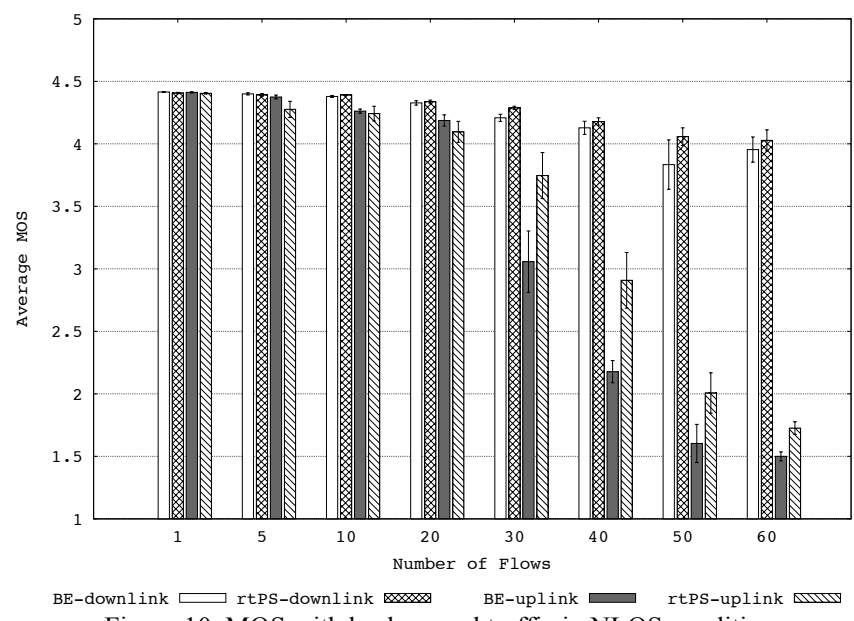

Figure 10. MOS with background traffic in NLOS conditions

Comparing the NLOS and LOS scenarios with background traffic, Figure 6 and Figure 10, the MOS values start decreasing at 20 simultaneous flows in LOS, while in NLOS it start deceasing at 5 flows. When comparing rtPS service class with BE, the rtPS supports up to 40 simultaneous flows in NLOS and 50 in LOS, while the BE in LOS supports up to 40 flows and in NLOS supports a maximum of 30 flows.

\section{Evaluation Summary}

This analysis demonstrated the efficiency of the WiMAX QoS mechanisms. It has been shown that the rtPS service class always has a better performance than BE, although in LOS conditions this difference is barely noted. The differences between rtPS and BE can result in less 20 simultaneous flows well sustained.

The results also show the benefits from access to the network in LOS when compared with NLOS conditions. This is an expected result, since the NLOS scenario offers much more interferences than the LOS scenario. Mostly due to the low USB CPE transmission power, leading to worse Signal-toNoise-Ratio (SNR) values, and the factors that arise in NLOS scenarios, such as multi-path fading and shadowing, the NLOS values are worse than in LOS.

The rtPS service class, without background traffic, supports the same amount of flows either in LOS or NLOS (50 simultaneous flows), while with background traffic it decreases from 50 simultaneous flows in LOS to 40 flows in NLOS. The BE class without background can support up to 50 flows in LOS and 40 flows in NLOS. With background traffic, this number decreases to 40 flows in LOS and to 30 flows in NLOS.

These results clearly depict the differences between LOS and NLOS, with and without background traffic and the importance of the correct service classes employment.

The impact of background traffic in the end-user QoE is noticeable in both LOS and NLOS access scenarios, resulting in the decreasing of entire simultaneous flows well supported.

\section{CONCLUSION}

The empirical assessment of the $4 \mathrm{G}$ and beyond broadband wireless networks, such as WiMAX, is very important since it allows the real evaluation of these technologies in real deployment conditions. Together with the assess of the network related parameters, such as delay or packet loss, also the end-user perceived quality should be considered, especially when transmitting multimedia application, such as VoIP or Video Streaming. This work provides an evaluation, in a real urban deployment, of the WiMAX technology capabilities of supporting multi-user VoIP calls in real access locations and environments, such as LOS and NLOS. Since Base Station equipment is able to support several simultaneous users, it is also relevant to observe the impact of other random background traffic in the QoE of the established VoIP calls.

This performed analysis showed that the changes in the Line of Sight could cause a large impact on the number of supported simultaneous flows, decreasing the average enduser perceived quality. In NLOS conditions, mostly because of the impact of multi-path fading and also due to the USB CPE limited transmission power, the support for multiple users is lower. The CPE transmission power affects the signal-to-noise ratio, causing a lower signal quality, which leads to a lower available bandwidth than in LOS scenario.

The background traffic has a large impact on the end-user VoIP QoE, since it will obstruct the channel with a random traffic, requiring the need of prioritization in the BS scheduling mechanisms. The different WiMAX QoS service classes, as evaluated in this work, support this traffic prioritization, allowing more simultaneous users with higher MOS values. The QoS service classes used were rtPS and BE, as the rtPS class guarantees the maximum OWD and the reserved rate, while the BE class does not offer any QoS guarantee. Because of these guarantees, it is shown that the rtPS has some advantages of maintain higher QoE levels, as well as the support for more simultaneous users. This service class can achieve higher MOS values with background traffic 
than $\mathrm{BE}$. The BE service class allowed to maintain good QoE levels in LOS without background traffic, where the BS scheduling algorithm cause some overhead to the rtPS traffic, allowing the BE to achieve similar results. Although this VoIP tests were conducted in rtPS class, due to software and hardware limitations, the most suited mechanisms for VoIP are ertPS and UGS, as they guarantee the maximum allowed jitter, delay and the reserved rate.

By conducting this analysis in a city environment with a real testbed and with similar end-user equipment as the network providers (i.e., USB CPEs), it was possible to provide a good and realistic assessment of a real world communications scenario.

\section{ACKNOWLEDGMENT}

This work was partially supported by EDPWiMAX project, funded by EDP Distribuição S.A., by the Portuguese National Foundation for Science and Technology (FCT) thought a Doctoral Grant (SFRH/BD/66181/2009) and by the CoFIMOM project PTDC/EIAEIA/116173/2009.

\section{REFERENCES}

[1] Ustafsson, E. V. A. G., Onsson, A. N. J., \& Esearch, E. R. R. (2003). Always Best Connected. IEEE Wireless Communications, (February), 49-55.

[2] 3GPP, "TS 21.101 - Technical Specifications and Technical Reports for a UTRAN-based 3GPP system (Release 8)," 2011.

[3] IEEE, "IEEE Standard for Local and metropolitan area networks - Part 16: Air Interface for Fixed and Mobile Broadband Wireless Access Systems," IEEE Standard, no. May, 2009.

[4] IEEE, "IEEE Standard for Local and metropolitan area networks - Part 16: Air Interface for Fixed and Mobile Broadband Wireless Access Systems,", IEEE Standard, 2004.

[5] IEEE, IEEE Standard for Local and metropolitan area networks - Part 16: Air Interface for Fixed and Mobile Broadband Wireless Access Systems, vol. 2005, no. February. 2005.

[6] Oh, S.; Kim, J.; , "User-perceived QoS performance enhancement for VoIP services in IEEE 802.16 systems," Pervasive Computing and Communications Workshops (PERCOM Workshops), 2010 8th IEEE International Conference on, vol., no., pp.570-574, March 292010 April 22010 doi: 10.1109 / PERCOMW . 2010.5470502.

[7] OPNET. Last Visit: 10/02/2012. Available: http://www.opnet.com/
[8] Zhang, H.; Mohammed, B.; Hang, N.; Xia, L.; , "Field performance evaluation of VoIP in 4G trials," Multimedia and Expo (ICME), 2011 IEEE International Conference on, vol., no., pp.1-4, 11-15 July 2011 doi: 10.1109 / ICME.2011.6011962

[9] Jadhav, S., Zhang, H., \& Huang, Z. (2011). Performance Evaluation of Quality of VoIP in WiMAX and UMTS. 2011 12th International Conference on Parallel and Distributed Computing, Applications and Technologies, 375-380. Ieee. doi:10.1109/PDCAT.2011.67.

[10] Durantini, A., Petracca, M., \& Ananasso, F. (2008). Experimental Evaluation of IEEE 802.16 WiMAX Performances at $2.5 \mathrm{GHz}$ Band. 2008 International Wireless Communications and Mobile Computing Conference, 338-343. Ieee. doi:10.1109/IWCMC.2008.59

[11] Bernardo, V., Sousa, B., \& Curado, M. (2009). VoIP over WiMAX: Quality of experience evaluation. 2009 IEEE Symposium on Computers and Communications, 42-47. Ieee. doi:10.1109/ISCC.2009.5202392

[12] Etemad, K. (2008). Overview of mobile WiMAX technology and evolution. IEEE Communications Magazine, 46(10), 31-40. doi:10.1109/MCOM.2008.4644117

[13] Alessio Botta, Alberto Dainotti, Antonio Pescapè, "Multi-protocol and multi-platform traffic generation and measurement", INFOCOM 2007 DEMO Session, May 2007, Anchorage (Alaska, USA)

[14] IEEE. (2008). RTP Payload Format for ITU-T Recommendation G.711.1 (pp. 1-15).

[15] ITU, T. S. S. of. (2003). Recommendation Y. 1541-Network Performance Objectives for IP-Based Services. Network.

[16] WiMAX Forum (2008). WiMAX system evaluation methodology v2.1. WiMAX Forum, Jan (pp. 1-209).

[17] IPerf. Last Visit: 10/02/2012. Available: http://dast.nlanr.net/Projects/Iperf

[18] ITU-T, "P.862 - Perceptual evaluation of speech quality (PESQ): An objective method for end-to-end speech quality assessment of narrowband telephone networks and speech codecs," 2001.

[19] ITU-T, "ITU-R BS . 1387-1 - Method for objective measurements of perceived audio quality (PEAQ)" 2001.

[20] ITU, T. S. S. of. (2009). ITU-T Rec. G.107 - The E-model: a computational model for use in transmission planning. Networks

[21] Cole, R. G. and Rosenbluth, J. H. 2001. Voice over IP performance monitoring. SIGCOMM Comput. Commun. Rev. 31, 2 (April 2001), 924.DOI $=10.1145 / 505666.505669$.

[22] Zhang, H.; Gu, Z.; Tian, Z.; , "QoS evaluation based on extend E-Model in VoIP," Advanced Communication Technology (ICACT), 2011 13th International Conference on, vol., no., pp.852-854, 13-16 Feb. 2011.

[23] Bernardo, V., Pentikousis, K., Jarno, P., Piri, E., \& Curado, M. (2009). Multi-client video streaming over wirelessMAN-OFDMA. Proceedings of the 4th ACM workshop on Performance monitoring and measurement of heterogeneous wireless and wired networks (pp. 46-53). ACM. 